\author{
For the period \\ March 15, 1991 through March 15, 1992 \\ For the grant
}

HIGH SPECTRAL RESOLUTION MEASUREMENTS

FOR THE

\title{
ARM PROGRAM
}

Henry E. Revercomb, Principal Investigator (608) 263-6758

Fred A. Best, Program Manager (608) 263-6777

Prepared for the

\author{
DEPARTMENT OF ENERGY \\ OFFICE OF ENERGY RESEARCH
}

Prepared by the

UNIVERSITY OF WISCONSIN

SPACE SCIENCE AND ENGINEERING CENTER

MAY 22, 1992 


\subsection{INTRODUCTION}

The DOE grant to the University of Wisconsin (UW) teamed with the University of Denver (UD) for the design and fabrication of high spectral resolution FTIR (Fourier Transform Infrared) instrumentation for the CART sites of the Atmospheric Radiation Measurement (ARM) Program began on September 15, 1990. The Year-one progress report covered the period from this beginning date to March 15, 1991. This year-two report covers the period from March 15, 1991 to March 15, 1992. The project is currently on or ahead of schedule for the most critical tasks that were defined for years one and two.

The ultimate objective of this grant is to develop three different types of instruments, named the AERI, AERI-X, and SORTI. The Atmospheric Emitted Radiance Interferometer (AERI) is the simplest. It will be available for early deployment at the first ARM site and will be deployable at several locations in the extended network to give horizontal coverage. The AERI will be an $0.5 \mathrm{~cm}^{-1}$ resolution (unapodized) instrument, which measures accurately calibrated radiance spectra for radiation studies and for remote sensing of atmospheric state variables. It would also be feasible to design an AERI for an aircraft or tethered balloon platform, although this is not a part of the current grant.

The AERI-X and the SORTI are higher spectral resolution instruments for obtaining the highest practical resolution for spectros sopy at the ARM central sites. The AERI-X, like the AERI, will measure atmospheric emitted radiance, but with resolutions as high as 0.1 $\mathrm{cm}^{-1}$. The Solar Radiance Transmission Interferometer (SORTI) will $\mathrm{n}$ easure the total transmission of the atmosphere by tracking the sun through changes in atmospheric air mass. The large solar signal makes it practical for this instrument to offer the ultimate in spectral resolution, about $0.002 \mathrm{~cm}^{-1}$.

This report includes the progress of the whole program, including both the UW and the UD activities. The UW has the primary responsibility for the AERI and the UD for the higher resolution instruments. However, many of the considerations for data system design, data format, software design, and calibration hardware are common. Coordination of activities in these areas is considered to be very important and will be receiving continuing attention now that UW has evaluated its prototype system, and UD has entered into its hardware design and implementation phase.

As described in detail below, there has been considerable progress made since the last progress reporting period. In general, the second year activities outlined in the original proposal are on or ahead of schedule. In the case of the AERI, development was accelerated in order to demonstrate instrument performance during the SPECTRE / FIRE II field experiments, and later during the STORMFEST field experiment. During both of these field trips, the AERI performed exceptionally well, on a nearly continuous basis.

\subsection{GENERAL SUMMARY OF PROGRESS}

In year 2, the AERI instrument was designed, fabricated, and successfully tested in the field in a "CART-like" environment, during both the SPECTRE and STORMFEST field experiments. The first field demonstration of AERI performance was originally to occur at the CART site in year 3. However, because of the timing of the two major field experiments in year 2, the AERI development was accelerated, thus allowing for a realistic setting for complete instrument testing. Testing at UD was conducted to determine a suitable interferometer for the SORTI instrument. A Bruker Model was chosen and has been ordered. Testing by UD at the FIRE experiment was performed on an AERI type 
instrument to determine calibration schemes that would be required for the AERI-X instrument.

\subsection{AERI Progress}

Most of the AERI subsystem concepts were verified in year one using the existing "Baby" HIS instrument. The efforts of year two can be broken into three areas: 1) refinement of subsystem design; 2), fabrication and field test of the AERI instrument; and 3) enhanced developments based on field test experience.

\subsubsection{Refinement of Subsystems Design}

The design of the calibration blackbodies was an important area of activity. An existing blackbody cavity (but undersized for the AERI application) was characterized thermally, with the test data then used to support a modeling effort that made use of a finite element heat transfer program. Using a scaled up version of this model, various geometries were investigated for the AERI blackbody, with the goal to minimize temperature gradients within the cavity. After an optimized design was determined, the cavities were fabricated and assembled. "Ultra-stable" thermistors were instalıd in these cavities and a careful temperature calibration was then carried out on each blackbody. Calibration accuracies of $\pm 0.03^{\circ} \mathrm{C}$ were obtained.

The Environmental Monitoring System also received careful design considerations. Bridge resistors were carefully chosen to optimize temperature readout :iensitivity and to minimize contributions to drift due to system temperature changes. In order to provide an indication of electronics drift or change in calibration, dedicated channels in the Environmental Monitoring System continually read precision resistors. These readings are fed into the data system along with all the other housekeeping data. The stability of these resistor readings is an indication of the stability of the electronics system.

Several software modifications vere required to upgrade the "Baby" HIS software to the AERI system. These modifications were driven by upgraded hardware including: the interferometer detector subsystems (two channel for AERI vs. one for the "Baby" HIS); the Environmental Monitoring System (16 channels for the AERI vs. 8 for the "Baby" HIS); and the scene switching mirror assembly (new motor and controller with integrated encoder for the AERI).

Great care went into the AERI optical alignment; elements that require alignment are the interferometer, scene switching mirror, and the blackbodies. A detailed procedure using specially designed alignment tooling was devised and carried out successfully on the AERI. This procedure uses the directly mapped field of view of the interferometer to establish the AERI system optical axis.

The AERI packaging and environmental control was another significant design effort. A split configuration (optics and electronics) was decided upon in which the optical components including the interferometer, scene mirror assembly, and blackbodies are structurally tied to a rigid optical bench which is shock mounted to the top surface of the cabinet containing the system electronics. This cabinet enclosure houses the ingest computer, blackbody controllers, and Environmental Monitoring System. The shock mounted optica! section has a thermally insulated cover with a viewing port. The electronics cabinet is thermally insulated and contains a internal fan to provide an isothermal interior temperature, and a thermostaticaly controlled fan that forces outside air into the enclosure when temperatures rise above a preset limit. 


\subsubsection{Fabrication and Field Testing of the AERI}

All the subsystems of the AFKI were tabricated, assembled and (the optical section) aligned by early November 1991. The system was then run side-by-side at UW with the "Baby" HIS, with the results indicating good agreement between the spectra generated by each instrument. The AERI was then brought to Coffeyville, KS to participate in the four week duration SPECTRE / FIRE II field experiments. On several cacasions the AERI operated simultaneously with the "Baby" HIS instrument, again with excellent correlation between instruments (see Figure 1).

\section{SPECTRE, $11-24-91$}
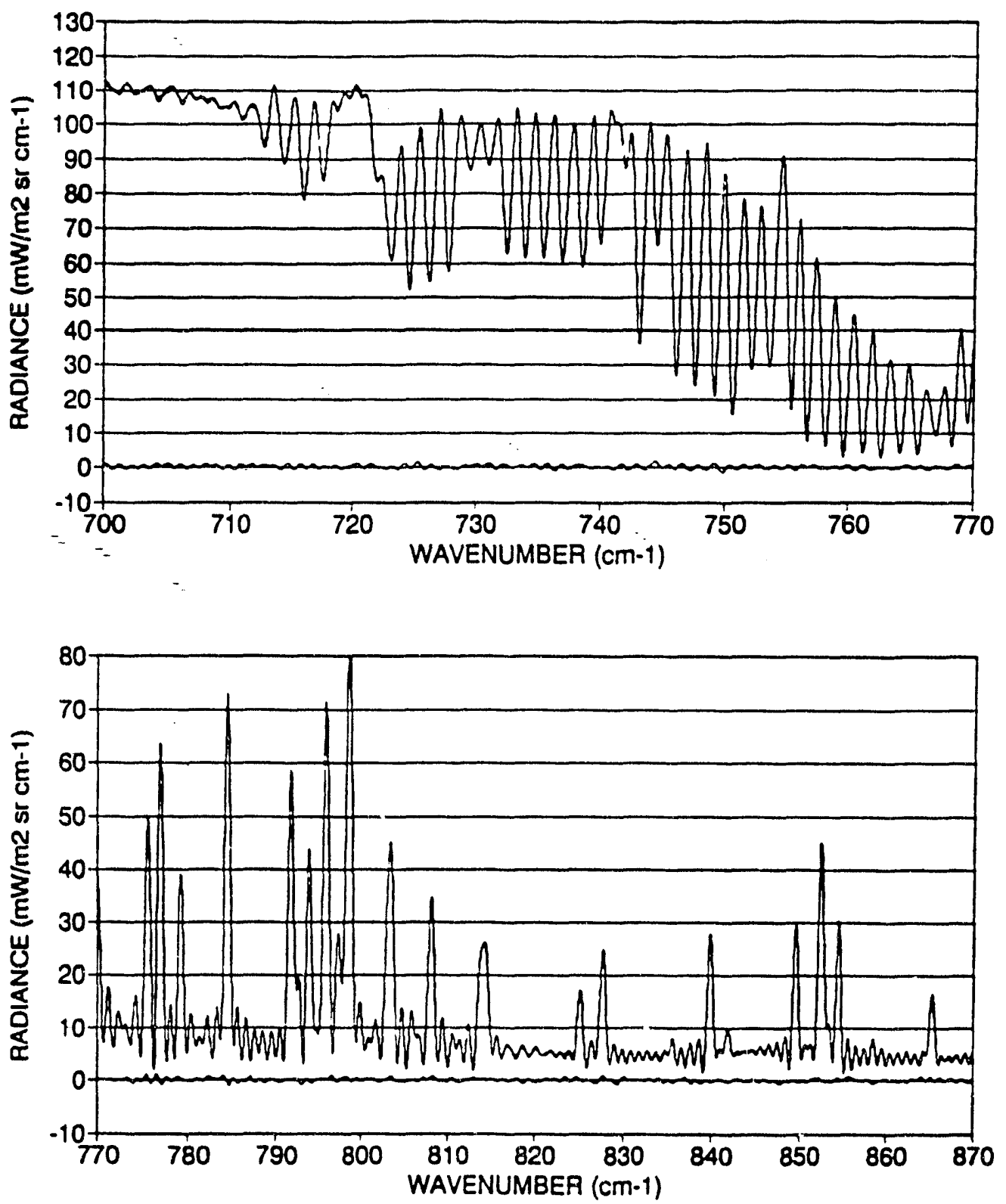

Figure 1 Comparison of spectra between "Baby HIS" and AERI at Coffeyville, KS. Effective blackbody emissivity was adjusted to compensate for small non-linear differences not yet accounted for. The near zero difference is close to the combined noise levels. 
In February of 1992 the AERI was deployed at Seneca, KS to participate in the six week duration STORMFEST experiment. This deployment provided an opportunity to exercise an automated software configuration. The AERI ran nearly 24 hours a day for the entire six weeks. Table 1 summarizes the AERI operations during this period.

Table 1 Summary of AERI operation hours in the field.

SPECTRE Coffeyville, KS (Nov. 11 through Dec. 7, 1991)

- 1278 Spectra (256 hours of operation)

- $40 \%$ operating time (10.7 days of operation / 27 day experiment)

STORMFEST Seneca, KS (Feb. 1 through Mar. 15, 1992)

- 4115 Spectra (823 hours of operation)

- $78 \%$ operating time (34.3 days of operation / 44 day experiment)

Given the AERI's rigorous operational experience, we feel that it is ready to participate in the integration and test phase at the CART site. However, it should be pointed out that there are several areas where further investigation can significantly enhance the AERI performance. For this reason it is highly desirable to have access to the AERI or an equivalent instrument for use as a test bed. The last section of this report provides progress details of each subsystem in the AERI. Areas where enhancements are envisioned are called out where appropriate.

\subsubsection{Enhanced Developments}

Several areas of development have proceeded in parallel with the AERI prototype fabrication and testing. These include an analysis of polarization sensitivity, characterization of system nonlinearities, operating system software enhancements, and integration of prototype code for retrieval of temperature and moisture vertical profiles in real time.

Laboratory experiments were carried out with a pair of infrared polarizing filters to examine the AERI sensitivity to incident polarized light. The effect on the overall calibration of sky data using blackbodies viewed with a scene mirror at several angles about the optical axis was a particular focus of the investigations. A significant polarization sensitivity due to the presence of the $\mathrm{KBr}$ beamsplitter at 45 degrees from the optic axis was quantified. The analysis showed that an aluminum scere mirror with silicon monoxide overcoating created unacceptable errors in the calibration, but a gold scene mirror which requires no overcoating reduced the polarization effects on calibration to an acceptably small value. The biggest difference occurs betweeen 8 and 10 microns where silicon monoxide has significant absorption. A gold mirror was acquired and installed in the AERI prototype before the SPECTRE/FIRE-II field program began.

The AERI system uses two detectors in the measurement of spectral infrared radiation in the 550 to $3000 \mathrm{~cm}^{-1}$ region. The spectral region is divided into two bands, a longwave band (550 to $1800 \mathrm{~cm}^{-1}$ ) using an MCT detector and a "shortwave" band (1750 to 3000 $\mathrm{cm}^{-1}$ ) using an InSb detector. The two detectors are in a "sandwich" configuration inside a single liquid nitrogen dewar with the same instrument field of view. The InSb detector is known to be inherently linear in its response to incident radiation while the MCT detector exhibits some nonlinear response. A program of testing and analysis was begun to 
determine the size and character of the nonlinearities and to develop an algorithm for realtime correction for nonlinear effects on the radiance calibration. During the period of this report significant progress has been made on understanding the nature of the effects and developing a model to empirically determine correction coefficients. Comparison of the AERI results to those of the HIS_aircraft instrument, which was designed to be highly linear, will be used for verification. Activity in this area will continue through implementation of correction algorithms at the CART site.

The operational system software for the AERI system has undergone several enhancements during the period of Nov. 1991 through March 1992. During the SPECTRE experiment an improved user interface was devised to facilitate instrument operations by previously untrained operators. Real-time feedback on instrument operations was also made a part of the operational system through a screen display of the current time and scene mirror position and the most recent raw and calibrated spectra. True concurrent data processing for real-time data calibration was implemented for the first time as well. The overall system software was thoroughly tested during intensive operating periods, several lasting 24-48 hours. The next major advance in operational software was made during the STORMFEST experiment (Feb. 1 - Mar 15, 1992) where the AERI system was operated for 44 days of continuous 24 hour operations with shutdown periods only during precipitation. In addition to the 24 hour operations, the prototype code for the real-time retrieval of temperature and moisture profiles from the calibrated radiance was also implemented and run on a 24 hour basis. This code (developed under a separate DOE ARM grant by Dr. W.L. Smith) will be implemented in future versions of the AERI system, in particular at the remote sensing boundary sites. The operations at STORMFEST demonstrated the excellent performance of the AERI prototype system under realistic operating conditions.

\subsection{AERI-X and SORTI Progress}

The basic FTIR instrument for the SORTI was ordered, and delivery is expected in May. This systern will have two detectors (InSb for 2 to 5 microns, and MCT for 5 to 14 microns). The instrument has a maximum path difference of $258 \mathrm{~cm}$, corresponding to a maximum theoretical resolution of $0.0025 \mathrm{~cm}-1$. The initial operation of the instrument in Oklahoma will be with fixed (not computer controlled) filters covering 7 to 14 microns, and 3 to 4 microns. Other regions will be evaluated for usefulness and resolution requirements. The detectors will also require liquid nitrogen fills every day.

The FIRE experiment provided an opportunity to operate an AERI type instrument in an AERI-X calibration mode. Specifically, calibrations were carried out at about half-hourly intervals. The instrument was operated outside in a small shipping box. The results indicate that thermal stability of the instrument was sufficient when interpolation of the instrument zero level was used. This is adequate for most of the observing periods. Since AERI-X will be operated in an office environment, a minimum of temperature control, more frequent calibration cycies, and interpolation of the zero level should be sufficient to provide good quality data.

\subsection{PROGRESS ON SPECIFIC TASKS}

This section provides further details of progress on individual subtasks. The subtasks listed below were originally defined in the budget breakdown that was provided to DOE as part of the "Budget Explanation" which was submitted as "Additional Information" to our original proposal. 


\subsection{Design Concept Verification}

\subsubsection{Test Cooling Options for Detectors}

UD has procured and tested a Sterling cooler on an interferometer system and found that excessive vibration produced by the cooler would not allow the interferometer to operate properly. Further investigations will continue, including tests on a interferometer with a linear drive, and general discussions with Bomem about mechanical cooler compatibility with interferometers. Bomem has recently delivered an interferometer system with a Sterling cooler to Fort Belvoir. Initial tests of this system show vibration immunity to the cooler reciprocal motion. It appears that compatibility problems between Sterling coolers and interferometers are starting to be solved. We still find the lifetime-to-cost ratio unattractive. Guaranteed lifetimes of 2,000 hours still run in the neighborhood of $\$ 7,000$.

Until the lifetime-to-cost ratio improves we feel the best approach for long term unattended detector cooling is either with a Joule-Thompson device which derives its cooling capacity by continuously expanding high pressure gas, or an auto-fill system which automatically fills the detector dewar with LN2 from a large storage vessel. Currently the auto-fill system looks most attractive to us.

\subsubsection{Hot / Ambient Calibration}

The AERI instrument makes use of the Hot / Ambient calibration scheme that was verified 'ssing the "Baby" HIS instrument in year one. The AERI also has the capability to view an LN2 blackbody. This capability is used only periodically to update the parameterization of the MCT detector non-linearity.

\subsubsection{Linearity Tests-Variable Temperature Blackbodies}

MCT detector nonlinearities on the order of 1 to $2 \%$ have been measured on our AERI instrument. This nonlinearity has been modeled and software has been written to correct for it. This software has yet to be implemented in the AERI on an operational basis. Further investigations and testing will be conducted to better understand the physics behind the nonlinearity, to possibly identify better correction schemes.

\subsubsection{Shortwave Detector (InSb) Test}

The AERI instrument has a "transparent" InSb detector mounted in front of the MCT detector, thus, both detectors simultaneously see the same incident radiation (in their respective sensing bands). Because of its inherent linearity, the InSb detector has proven to be very useful in helping to understand and correct for the MCT nonlinearity. The InSb detector is expected to be valuable in both humid environments such as the Western Tropical Pacific Site, and for use in cloud radiance studies.

\subsubsection{Closed Cycle Sterling Cooler}

The UW has no current plans to purchase a Sterling Cooler. UD is investigating this technology for use in detector cooling, but has not been successful in finding a device that is compatible with their interferometer. Sterling Cooler technology is improving rapidly and lifetime-to-cost ratios are improving constantly. When this option looks more attractive we may wish to pursue our own test program; however, our current baseline for long term detector cooling for the AERI, will be to use a relatively inexpensive and reliable liquid nitrogen auto-fill system.

\subsubsection{Coordination Meetings With UD}

With UD firming up system concepts for the AERI-X and with the AERI system design completed, possible overlaps in hardware and software will be decided upon in the near future. Candidate areas where the AERI-X can make use of AERI developments are: calibration blackbodies and controllers; detector cooling scheme; scene mirror system; A/D 
sysitem; calibration and display software; networking scheme; and data formats. UW has provided details of each of these subsystem: to UD. A meeting will be held this summer (1992) to firm up which subsystem designs will be used by UD and how they will be implemented within the AERI-X.

\subsection{Subsystem Design, Fabrication, and Testing}

\section{3,2.1 Two Channel Interferometer}

A two channel (InSb and MCT) Bomem MB100 has been delivered, checked out and integrated into the AERI system. The interferometer has operated flawlessly since we received it in April of 1991. The two channel option (as opposed to MCT only) is a feature we think is worth including in all the AERI instruments (see section 3.1).

\subsubsection{Two Detector Control Software}

We received the Bomem software that is used to acquire unapodized spectra. During the evaluation testing of this software we found and corrected an error. We suggested that an enhanced feature be included in the software, that would compute the variance of the magnitudes of the spectra vs. wavenumber during a dwell. This "variance software" has been delivered and integrated into the AERI. This feature is used to flag a situation were the scene radiance changes during a dwell of the sky because of the movement of clouds.

\subsubsection{Data Management and LAN Subsystem}

The hardware and software for the acquisition computer along with the Novell network has been successfully integrated and tested in the AERI. This configuration was exercised extensively in the field with no major problems being identified. An improved scheme using a TCPIP ethernet network is presently being investigated. This system will eliminate the server computer and make our system more compatible with the CART site protocols.

\subsubsection{Environmental Control and Packaging : Considerations}

The scheme adopted for the AERI assumes that it will be placed in a waterproof shelter with a zenith view port (that can be covered). The AERI will operate in this shelter when the temperature is within the range -20 to $35^{\circ} \mathrm{C}$. The AERI is split into two parts. The interferometer / front end optics and the electronics. All the components of the interferometer / front end optics, which includes the blackbody cavities and the scene mirror, are structurally tied together and shock mounted to the top surface of the cabinet that houses the electronics. This whole assembly (the AERI) is mounted on heavy duty wheels with brakes. The interferometer / front end optics section is covered with a thermally insulated cover that contains a zenith viewport. Heat generated by the interferometer keeps the local operating environment of this section well within the operating limits of the interferometer. The electronics cabinet is also thermally insulated and contains the data ingest computer, cavity controllers, and the A/D system. There is a fan locaied inside the cabinet that is designed to keep the local environment isothermal (by mixing the air within the cabinet). This fan is always operating. Another fan located in the floor of the cabinet is thermostaticly controlled to introduce outside air when temperatures inside the cabinet enclosure rises above a preset value. The above described configuration worked with good results in Coffeyville, KS during SPECTRE (in November of 91) where the ambient temperatures of the shelter were between $-5^{\circ} \mathrm{C}$ and $25^{\circ} \mathrm{C}$, and also during the STORMFEST field experiment (in February of 92) where shelter temperatures were between $-15^{\circ} \mathrm{C}$ and $15^{\circ} \mathrm{C}$.

At the CART site, the AERI will be placed inside a trailer that will be environmentally controlled to a comfortable "shirtsleeves" environment. At the time of initial deployment, the AERI will look through a zenith port in the roof of the trailer. There will be a "boot" that interfaces this viewing port to the viewport in the cover of the AERI. This 
configuration leads to an easy interface to the trailer and has no impact on the existing AERI configuration. It is desirable to have the front end optics (scene mirror and ambient blackbody) at close to outside ambient temperature. A scheme will be investigated that allows the AERI front end optics to protrude through the wall of the trailer using an insulated flexible collar as a thermal interface between the AERI and the trailer wall. The part of the AERI that protrudes the wall will be thermally isolated from the trailer and hence operate at outside ambient temperature. To protect the protruding hardware during nonoperating weather, it is envisioned that a bay window type arrangement with a zenith hatch could be used.

\subsubsection{Optics Bench and Support Table}

The optics bench of the prototype AERI is the mounting platform for the interferometer, calibration blackbody cavities, and scene switching mimror. For the prototype, this structure was designed to be very stiff and it includes a general blackbody mounting interface that will allow six positions for blackbody placement. The optics bench mounts to the electronic enclosure through four standard shock mounts. This optics bench design was deemed desirable for the prototype because it easily facilitated various hardware configurations for system testing purposes. Further investigations may lead to a simpler optics bench configuration for future AERI instruments that won't require configuration flexibility.

\subsubsection{Scene Mirror Motor and Controller}

The effects of polarization introduced into the AERI by the aluminized angled $45^{\circ}$ scene switching mirror, were tested using an infrared polarizer. These tests showed that polarization effects (that were unable to be calibrated out) were unacceptably high with this mirror. Published test data from first surface gold mirrors indicated acceptable polarization effects. A gold mirror was ordered and is now used in the AERI. The same series of polarization tests that were performed on the aluminized mirror have yet to be performed on the gold mirror. These tests, which will hopefully verify published polarization data for gold surface mirrors, are planned for the near future.

The $45^{\circ}$ scene mirror on the AERI prototype scene switching mirror assembly uses a Parker - Compumoter motor and matched controller with an integrated angular encoder for an absolute reference. The AERI control software was changed to accommodate this motor configuration and the system is performing very well. A future enhancement may include reading the encoder position at all times during operation so that the mirror position can be included in the data stream. This information could be used to set a flag in the event that the mirror position did not end up at its commanded location.

\subsubsection{Blackbody Calibration Sources}

An existing blackbody cavity that was part of the "Baby" HIS system (but is too small for the AERI application) was extensively tested to determine its equilibrium temperature field, temperature transient response, and power consumption under various control temperatures. From these test data, thermal resistances and capacitances for this cavity were calculated. A heat transfer finite element model of this cavity (including the air within the cavity) was then constructed and "tweaked" to agree with the test results. The geometrically similar but scaled up cavity (suitable for the AERD) was then modeled using the "tweaked" heat transfer coefficients. This finite element model was then iterated (critical dimensions were changed) several times, with the goa! to minimize cavity temperature gradients and also to predict power vs. control temperature, which was used to size the controller power supply. 
Four cavities with a configuration based on the finite element results were then machined, assembled, and calibrated. Two of these cavities were sent to UD to support ongoing tests there. The other two cavities are part of the AERI system, one is used as the Hot blackbody and one for the Ambient. The assembly of the cavities involved winding 200 turns of heater wire on the outside diameter of the cavity. A special winding tool was fabricated to facilitate this operation. There are four "super stable" YSI precision thermistors in each cavity. Each of these thermistors, after assembly into the cavities, was calibrated against an NIST traceable standard to within $0.05^{\circ} \mathrm{C}$. Three calibration points were used to determine a unique set of Steinhart-Hart coefficients for each thermistor. These coefficients are then incorporated in the software of the control software. Future enhancements of the cavity design may include a metal-to-metal interface at the aft end of the cone for easy periodic calibration in-the-field using a calibrated standard.

Each of the four blackbodies that were manufactured has a dedicated electronic controller. The controller design was based on improvements made to an existing system at the UW. The controller circuit board was laid-out and manufactured, populated, assembled and tested in-house. The controller design allows 900 different control temperatures above ambient up to $60^{\circ} \mathrm{C}$. The temperature stability of the of blackbody / controller combination has been determined to be a fraction of the absolute temperature calibration accuracy $( \pm 0.03)$ over a blackbody view period ( 120 seconds).

\subsubsection{Alignment Procedure and Tooling}

A very successful procedure (along with the associated tooling) was developed to align the AERI interferometer to the scene mirror and calibration blackbodies. The interferometer optical axis was established by mapping the field of view using a hot point source positioned incrementally at different locations on a grid within the viewing field. The reference axis of the grid was positioned on the autocollimated ray from the center of the interferometer window. Using the align function of the Bomem interferometer, which displays the interferogram peak in real time, the shape of the field of view was determined by plotting the interferogram peak at each grid location of the hot point source. This was done for both MCT and InSb channels. As it turned out, the field of view for both channels was remarkably centered on the autocollimated window center ray. This ray was then chosen as the optical axis of the interferometer.

To align the AERI system, an "optical axis tool" with two reticules that represent the scene mirror motor axis was installed in the motor position. An alignment scope was set up to view through the center of the two reticules looking in the direction of the interferometer front window (this was defined as the optical axis of the AERI system). Using the alignment scope the normal to the interferometer window center was then placed on the optical axis. This interferometer position was then blocked in such a way that it could be removed and repositioned reliably. The interferometer was then removed from the optics bench and the alignment scope was set up to view through the optical axis tool from the back side (viewing in the direction of a ray coming from the interferometer). The optical axis tool was removed and replaced by the scene mitor assembly, which holds the scene mirror at $45^{\circ}$ and provides rotational positioning to view the scene (zenith) or five other blackbody positions. The scene mirror was commanded, in turn, to each nominal blackbody' position. At each position the center of the blackbody aperture was placed on the axis defined by the alignment scope (which was reflected of the $45^{\circ}$ mirror to the aperture center). After the blackbodies were aligned, they were scribed with reference marks so that they could be removed and repositioned accurately. After both field experiments in which the AERI participated, the system was disassembled and the alignment was hecked, this time using a laser in place of the alignment scope. The alignment positions remained unchanged thus verifying long term staibility and verifying a slightly simpler alignment scheme (with a laser as opposed to an alignrnent scope). 


\section{3:2.9 Environmental Monitoring System}

The Environmental monitoring System consists of a 16 channel 16 bit analog to digital (A/D) converter and a signal conditioning system. Temperatures (thermistors) of both blackbodies and several system temperatures, as well as surface pressure and humidity are measured periodically by this system and fed into the AERI data stream. As a measure of system drift, some of the AVD channels are dedicated to continually reading fixed precision low temperature coefficient resistors that correspond to the thermistor resistances at the high and low range of the temperatures expected. To help minimize drift problems, matched precision low temperature coefficient resistors were installed into the signal conditioning bridges. The resistor values were chosen to optimize temperature readout sensitivity. A semi-automated calibration scheme was designed and successfully implemented into the Environmental Monitoring System. This calibration procedure can be performed periodically as required. It involves having each channel read four different precision resistors and then deriving a best fit calibration line through this data. The slope and intercept of this line are stored and used automatically in the software for each channel. These two calibration coefficients, along with the three Stienhart and Hart coefficients that were determined for each thermistor using a three point temperature calibration, uniquely define the end-to-end calibration of the A/D channel. Future enhancements of this system may include a more simplified calibration procedure.

\subsubsection{Noise Testing and Gain Adjustments to Interferometer}

As an initial checkout of the AERI system, responsivity and noise tests were performed, and the detector preamp gains were adjusted to avoid signal saturation and to optimize the signal-to-noise ratio.

\subsubsection{Integrated System Software}

In order to expend a minimum of effort and to end up with a high probability of success, the AERI integrated system software used the existing "Baby" HIS system software as a foundation. Software modules were changed to accommodate the two channel interferometer, new scene motor controller, and new data acquisition system. The system software for the AERI has been shown to work well during long term operation in the field. Although this software has served us well to date, it was not built from the top down and thus suffers from a lack of flexibility. Now that we have a comprehensive understanding of the AERI system, a rewrite of the software at some point in the future will simplify the software support requirements as well as provide more flexible operating modes.

While in the field several enhancements in the operational software were written and implemented (see section 2.1.3).

\subsubsection{Co-Aligned Video Imagery}

This option was not built into the AERI protolype. We have learned that a periodic coaligned video image of the scene in the data stream would be very desirable and plan to implement this feature as soon as time and funding allow.

\subsubsection{Develop Systems Users Manual}

The users manual has not been written. We view this as a very important task that we intend to carry out in the future. As we gain more experience with the AERI and as we learn more about the future users of the system, we will be better able to target a manual to the proper audience. 


\subsection{System Verification Tests}

After the AERI was assembled it was checked out to insure that all the subsystems were operating properly. Following these tests the AERI was tested along side with the "Baby" HIS instrument, with both instruments viewing the same sky scene. There was excellent correlation between the spectra obtained from these instruments. In addition, there were several coincident periods of observing by both these instruments at SPECTRE in Coffeyville, KS. Again the correlation between instruments was remarkable.

Comparison tests between the AERI and the HIS aircraft experiment have been impossible to conduct because either the aircraft experiment has been involved in repair work or it has been out supporting field experiments. We plan to conduct these intercomparison tests in the near future. 
DATE

FILMED

$12 / 11 / 92$ 
